\title{
ATRIÇÃO DE PARTÍCULAS ESFÉRICAS E IRREGULARES EM LEITO DE JORRO
}

\author{
R. C. SOUSA ${ }^{1}$, F. B. FREIRE ${ }^{1}$, J. T. FREIRE ${ }^{1 *}$ \\ ${ }^{1}$ Universidade Federal de São Carlos, Departamento de Engenharia Química \\ e-mail: freire@ufscar.br
}

\begin{abstract}
RESUMO
Dependendo do tipo de particulado e das condições operacionais utilizadas no leito de jorro, as partículas podem reduzir de tamanho e consequentemente de peso devido à atrição. Entretanto, alguns materiais podem ser resistentes e não se desgastarem. $O$ fato é que os efeitos da atrição influenciam diretamente nas características fluidodinâmicas do leito de jorro. Sendo assim, o presente estudo teve como finalidade analisar a cinética de atrição de materiais esféricos (vidro e alumina) e partículas de dolomita no leito de jorro convencional, bem como contribuir com o entendimento do fenômeno da atrição. Para isso, foram realizados ensaios experimentais e numéricos variando a velocidade do ar de entrada. Os resultados experimentais da cinética de atrição mostraram que tanto a massa das esferas de alumina quanto das partículas de dolomita reduziu linearmente ao longo do tempo e, além disso, foi observado, também, que a redução foi mais acentuada para a maior velocidade do ar de entrada. No que se refere à parte numérica, foram utilizados os modelos de primeira ordem propostos na literatura. Em relação ao desempenho do leito de jorro, a atrição dos materiais particulados influenciou na fluidodinâmica fazendo com que a velocidade de mínimo jorro se tornasse menor ao longo do tempo de operação.
\end{abstract}

\section{INTRODUÇÃO}

Nas indústrias químicas, agrícolas, farmacêuticas, entre outras, é inevitável o surgimento da atrição decorrente da movimentação do material particulado durante o processo. Como efeito, a atrição pode alterar as propriedades dos sólidos e tornar-se um sério problema capaz de influenciar na qualidade do produto final e no desempenho dos equipamentos (BENROSE e BRIDGWATER, 1987; PAPADOPOULOS, 1998).

Em processos que utilizam leitos móveis como equipamentos, a atrição é considerada um fenômeno de desgaste que ocorre quando partículas do material sólido são removidas, como consequiência, pode levar a redução de tamanho da fase particulada e a emissão de partículas finas (conhecido na literatura como "finos") que na grande maioria das vezes necessita ser separada ao final do processo (RAY e JIANG, 1987).

A atrição pode ocorrer de duas diferentes formas, uma conhecida como abrasão superficial, ou seja, quando há redução gradual do tamanho devido à quebra de partículas da superfície e a outra como fragmentação, que é a divisão do sólido em várias outras partes de tamanhos menores.

Contudo, ambas as formas dependem de fatores que incluem as propriedades dos materiais, como a porosidade, o tamanho, a dureza, a massa específica, a forma, entre outros. Assim como pode depender também das condições operacionais, como o tempo de residência, a velocidade do ar, a pressão e a temperatura (RAY e JIANG, 1987). 
Estudos têm mostrado que o mecanismo predominante no leito de jorro é a abrasão superficial. $O$ efeito é maior na região do jorro durante o transporte pneumático, quando os sólidos colidem entre si e contra a parede de sólidos que formam o canal do jorro. Pode ocorrer também na região da fonte, por impacto, isto é, quando os sólidos colidem uns contra os outros e contra as fronteiras físicas do leito de jorro (FERNÁNDEZAKARREGUI et al. 2012).

Entretanto, a abrasão superficial depende, além das propriedades dos materiais sólidos, de parâmetros fluidodinâmicos como o diâmetro do orifício de entrada do ar, o ângulo do cone, a altura do leito de partículas e a vazão do ar de entrada do leito (MATHUR E EPSTEIN, 1974).

São poucos os trabalhos encontrados na literatura que analisaram o efeito da atrição em função do tipo de material particulado e dos parâmetros fluidodinâmicos no leito de jorro. Da mesma forma sobre a modelagem matemática, os modelos encontrados na literatura foram utilizados para analisar a cinética de atrição em leitos fluidizados. A grande maioria dos modelos é constituída por uma equação de primeira ordem e foram aplicados para avaliar o desgaste do material sólido em leitos fluidizados.

O trabalho realizado por FernándezAkarregui et al. (2012) merece destaque por analisar, simultaneamente, dados experimentais e numéricos da cinética de atrição de dois tipos de areia em função da velocidade do ar e da temperatura. Os autores observaram que ambos os materiais perderam massa ao longo do tempo e a perda se tornava maior com o aumento da velocidade do ar.

Além disso, a cinética de atrição foi simulada a partir de correlações empíricas compostas por equações de primeira ordem propostas por Gwyn (1969) e Lee et al. (1993). Os autores concluíram que a aplicação desses modelos no leito de jorro foi satisfatória, uma vez que os resultados preditos apresentaram boas concordâncias com os experimentais.

Dentro deste contexto, o presente trabalho tem por finalidade analisar a atrição de diferentes tipos e geometrias de materiais particulados no leito de jorro convencional, bem como contribuir no entendimento do fenômeno da atrição. Para isso, foi analisada a cinética de atrição tanto do ponto de vista numérico quanto experimental, variando a velocidade do ar de entrada e, paralelamente, observar o efeito da atrição no desempenho fluidodinâmico do leito de jorro.

\section{MATERIAIS E MÉTODOS}

\subsection{Materiais Particulados}

Os materiais particulados utilizados na realização dos ensaios experimentais são: esferas de vidro, esferas de alumina e partículas de dolomita. A caracterização utilizada para identificar as propriedades físicas, quanto ao tamanho, porosidade $\mathrm{e}$ massa específica (real e aparente), bem como as técnicas de medidas utilizadas é apresentada na Tabela 1.

Tabela 1 - Propriedades dos materiais particulados.

Propriedade Vidro Alumina Dolomita

\begin{tabular}{cccc}
\hline $\mathrm{dp}(\mathrm{mm})$ & $\begin{array}{c}3,2 \\
\text { (a) }\end{array}$ & $\begin{array}{c}3,0 \\
\text { (a) }\end{array}$ & $\begin{array}{c}6,22 \\
\text { (b) }\end{array}$ \\
$\rho_{\text {ap }}\left(\mathrm{kg} / \mathrm{m}^{3}\right)$ & - & 1501 & - \\
& & (c) & - \\
$\rho_{\text {real }}\left(\mathrm{kg} / \mathrm{m}^{3}\right)$ & $\begin{array}{c}2500 \\
\text { (d) }\end{array}$ & $\begin{array}{c}2482 \\
\text { (c) }\end{array}$ & $\begin{array}{c}2819 \\
\text { (c) }\end{array}$ \\
& 1 & 1 & 0,85 \\
$\Phi(-)$ & (e) & (e) & (b) \\
\hline
\end{tabular}

(a) Peneiramento, (b) Análise de imagem, (c) Picnometria a gás, (d) Picnometria líquida (e) Fonte: Narimatsu (2001). 


\subsection{Equipamento e unidade experimental}

$O$ equipamento utilizado na condução dos ensaios experimentais foi um leito de jorro convencional do tipo cone-cilíndrico. $\mathrm{O}$ cone apresenta ângulo de $60^{\circ}, 20 \mathrm{~cm}$ de altura, diâmetro de entrada do ar igual a $3 \mathrm{~mm}$ e diâmetro superior de $30 \mathrm{~cm}$. O corpo cilíndrico apresenta $30 \mathrm{~cm}$ de diâmetro interno e 1,20 m de altura.

$\mathrm{Na}$ unidade experimental, como mostra a Figura 1, o leito de jorro está conectado a um soprador de 4 HP por tubulação de ferro galvanizado de duas polegadas, ao longo da linha há um sistema de desvio do ar para a entrada do leito de jorro (by-pass) constituído por válvulas gavetas de duas polegadas e medidor de vazão do ar tipo placa de orifício. As medidas de pressão foram realizadas na entrada do leito de jorro e na placa de orifício por transdutores de pressão com faixa de operação que variam de 0 a 5 PSI. Os transdutores estavam conectados a um sistema de aquisição composto por uma placa condicionadora de sinais, uma placa de aquisição de dados, um microcomputador e um software de aquisição desenvolvido em linguagem de programação gráfica LabVIEW.

Figura 1 - Diagrama esquemático da unidade experimental. Detalhes: $1-$ Soprador; 2 - By pass; 3 - placa de orifício; 4 - Sistema de aquecimento; 5 - termopar e transdutor de pressão; 6 - Condicionador de sinais; 7 - Computador.

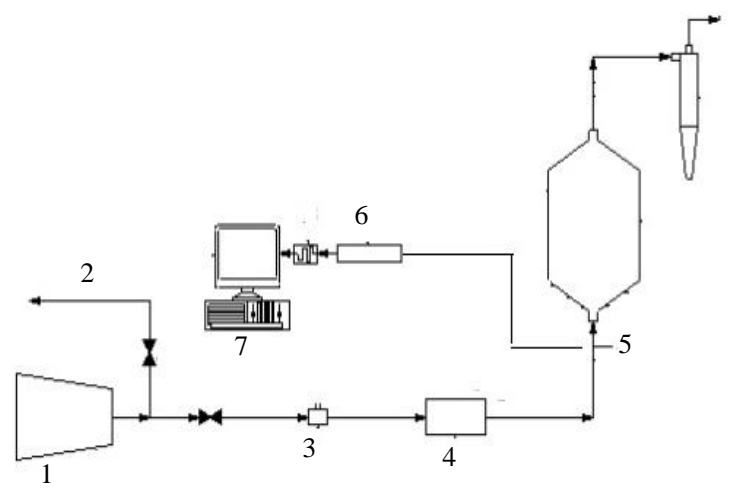

Fonte: Nascimento, 2013

\subsection{Procedimentos experimentais.}

Caracterização dos materiais sólidos: o peneiramento das esferas de vidro e de alumina foi realizado utilizando peneiras do tipo Tyler/Mesh com a seguinte série: 4, 5, 6, $7,8,10$ e 12 , sob agitação por um período de 30 minutos. Com os dados obtidos do peneiramento calculou-se o diâmetro médio a partir da equação de Sauter, mostrada na Equação 1, a qual considera a fração mássica retida em cada Mesh e o diâmetro médio entre as peneiras.

$$
d p_{s}=\frac{1}{\sum \frac{x_{i}}{\overline{d p_{i}}}}
$$

No caso das partículas de dolomita, o diâmetro de Ferret e a esfericidade foram determinados utilizando o software Image Pro Plus 4.5. Para isso, foi utilizada uma amostragem com 60 partículas de dolomita antes do ensaio de atrição.

A massa específica dos materiais particulados foi determinada através de picnometria líquida, neste caso utilizando água e picnometria gasosa, através de um picnometro a gás Hélio.

Obtenção das curvas fluidodinâmicas: inicialmente, com o leito de jorro carregado de partículas, o soprador era ligado e aguardava-se o sistema atingir o estado estacionario. Depois de atingido essa condição, as curvas fluidodinâmicas foram construídas como propõe Mathur e Epstein, (1974).

Obtenção dos perfis de cinética de atrição: depois de construída as curvas fluidodinamicas, a velocidade do ar foi fixada para uma condição de operação e mantida por um determinado intervalo de tempo. Ao final desse tempo, a válvula da tubulação de descarga do ar em excesso era totalmente aberta e o soprador desligado. Com o sistema totalmente desligado, foram 
retirados os materiais particulados pela região cônica do leito de jorro e em seguida pesados em uma balança digital com precisão de $0,001 \mathrm{~g}$. No caso das esferas de alumina, foi necessário realizar a secagem na estufa de convecção forçada por um período de 24 horas, devido a rápida adsorção de umidade durante o processo. Assim, ao final de cada ensaio de atrição as esferas de alumina foram secas e posteriormente pesadas.

Determinação da perda de massa $\left(X_{a}\right)$ : a massa perdida durante a atrição foi definida e quantificada de acordo com a Equação 2, sendo $M_{\mathrm{i}}$ a massa do sólido no tempo (t) e $M_{0}$ a massa inicial do sólido .

$$
X_{a}=1-\frac{M_{i}}{M_{0}} x 100
$$

Simulação dos modelos: a simulação numérica foi realizada utilizando os modelos de Gwyn (1969) e Lee et al. (1993) compostos pelas equações 3 e 4 , as quais são apresentadas na Tabela 2 .

Tabela 2 - Equações dos modelos de Gwyn (1969) e Lee et al. (1993).

$$
R t=k p m t^{m-1} W \quad \text { Gwyn (1969) }
$$

$W e=\left(W_{0}-W \min \right) e^{-k a t}+W \min$ Lee et al. (1993)

(4)

A constante $k_{a}$ foi obtida através do método adotado por Fernández-Akarregui et al. (2012), o qual considera a velocidade de mínimo jorro, como mostra a Equação 5.

$$
k_{a}=k_{0} e^{-k_{1} / u_{0}\left(u_{0}-u_{m j}\right)}
$$

Os parâmetros do modelo foram estimados com base em rotinas computacionais desenvolvidas no software Matlab. Para isso, tomou-se como critério a minimização dos quadrados dos desvios entre dados observados e preditos pelos modelos empregados nesse trabalho, utilizando a função lsqcurvefit.

\section{RESULTADOS E DISCUSSÃO}

Inicialmente, os ensaios experimentais da atrição referem-se às esferas de vidro e de alumina, utilizando como condições operacionais, a relação $\mathrm{u} / \mathrm{u}_{\mathrm{mj}}=1,10 \mathrm{e} \quad \mathrm{o}$ período de operação igual a 2250 minutos.

São apresentadas nas Figuras 2 e 3 curvas de distribuição granulométrica desses materiais referentes ao início e o fim dos experimentos. As curvas foram construídas a partir de dados da fração mássica retida em função do diâmetro de peneiras. Com esses dados o diâmetro médio do leito de partículas foi calculado e utilizado como parâmetro para identificar se houve redução de tamanho.

Observa-se na Figura 2, que os comportamentos referentes ao início e o fim são similares e se sobrepõem. Isso indica que para as condições operacionais utilizadas as esferas de vidro resistiram ao atrito no interior do leito de jorro e não se desgastaram, apresentando o tamanho médio igual a 3,17 mm de diâmetro.

Figura 2 - Curvas de distribuição granulométrica das esferas de vidro.

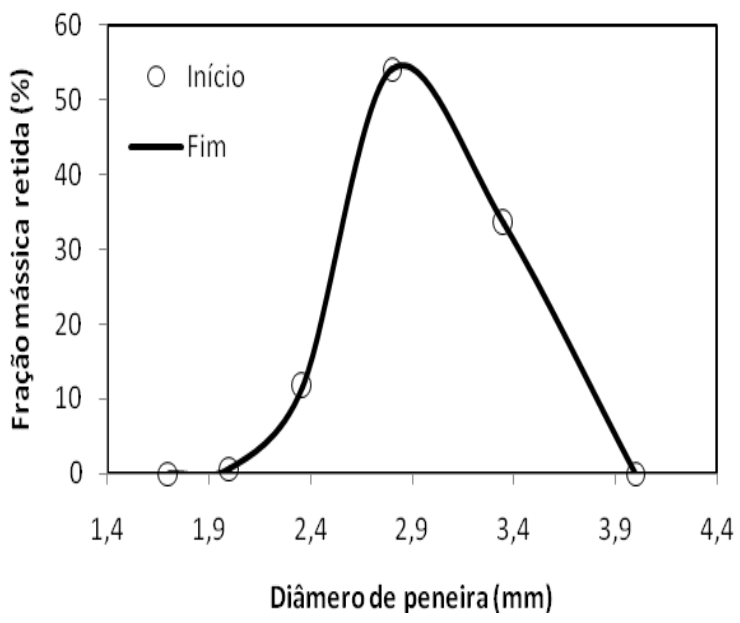


Por outro lado, ao analisar a distribuição de tamanho das esferas de alumina apresentada na Figura 3, verifica-se que as curvas são semelhantes, porém a curva granulométrica referente ao final do processo de atrição está deslocada para a esquerda. Visualmente é possível observar que houve redução de tamanho das esferas de alumina para a faixa de peneiras utilizadas, isto foi comprovado através do cálculo do diâmetro médio. Inicialmente as esferas de alumina apresentavam o diâmetro médio igual a 3,42 $\mathrm{mm}$ e após a atrição passaram a ter o diâmetro igual a 3,09 mm.

Outro fator a ser observado na Figura 3 é a redução acentuada das partículas maiores em relação às menores. Esse comportamento pode estar relacionado com o tamanho da partícula, pois segundo Lee et al. (1993), partículas pequenas contém menos falhas ou imperfeições em sua estrutura quando comparadas com partículas grandes e assim são mais resistentes ao atrito por impacto.

Figura 3 - Curvas de distribuição granulométrica das esferas de alumina.

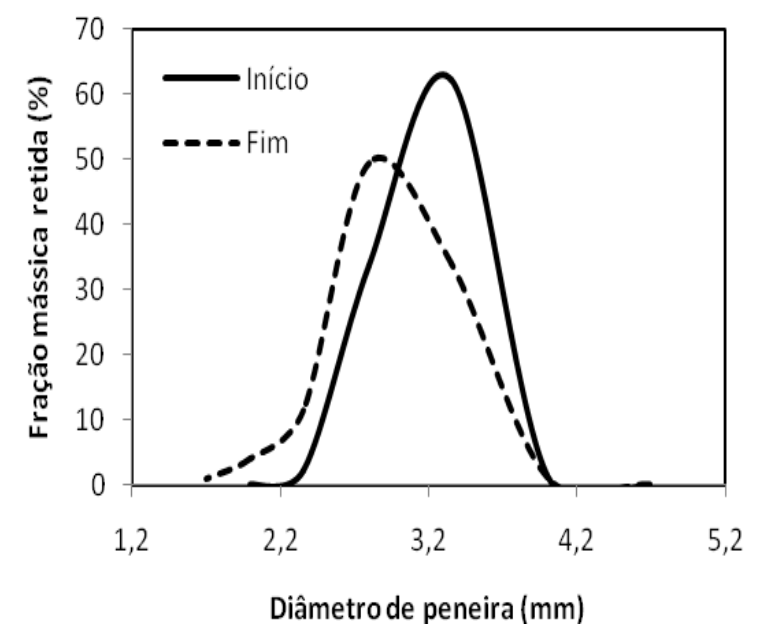

Cabe mencionar que o comportamento das curvas da distribuição granulométrica referente ao início e o fim da atrição apresentadas na Figura 3 é típico do fenômeno conhecido por abrasão superficial e comumente observado em processos que utilizam leitos fluidizados. Segundo Reppenhagen e Werther (2003) a abrasão superficial de materiais sólidos em leitos móveis acontece porque a superfície dos sólidos se quebra em partículas finas, fazendo com que se tornem menores que o tamanho original.

Uma vez observado que as esferas de alumina reduziram de tamanho devido o desgaste, realizou-se a análise dos dados da cinética de atrição e da perda de massa, como mostra a Figura 4. De maneira análoga, a análise foi feita também para as partículas de dolomita e apresentada na Figura 5.

Os ensaios foram realizados para os dois materiais particulados utilizando como condições operacionais a relação $\mathrm{u} / \mathrm{u}_{\mathrm{mj}}=1,10$, a massa inicial $\left(\mathrm{M}_{0}\right)$ igual a $2,58 \mathrm{~kg}$ e o tempo (t) igual a 2250 minutos para as esferas de alumina e 2430 minutos para as partículas de dolomita.

Através da cinética de atrição, verificam-se nas Figuras 4 e 5 que os comportamentos são similares para os dois tipos de materiais particulados, ou seja, a massa reduz linearmente em função do tempo. Linearidade esta constatada pelo valor do coeficiente de correlação, próximo da unidade. As reduções foram significativas se comparadas com a quantidade inicial, sendo que $o$ leito de alumina no final do experimento passou a pesar $1,75 \mathrm{~kg}$ e as partículas de dolomita $1,62 \mathrm{Kg}$.

Os comportamentos qualitativos apresentados nas Figuras 4 e 5 diferem com o que foi encontrado na literatura. Curvas de cinética de atrição observadas em leito de jorro e inclusive em leitos fluidizados mostram que a redução da massa tem um comportamento exponencial até atingir um dado intervalo de tempo, a partir disso, sua variação não é mais significativa e inicia-se 
um patamar que pode ser considerado estacionário.

Possivelmente, o fato da cinética de atrição dos materiais terem sido lineares pode ser justificado pelo tempo de experimento, o qual não foi suficiente para que o estado estacionário fosse atingido. Além disso, cabe ressaltar que o tempo utilizado em vários trabalhos vistos na literatura é maior quando comparados com que foi utilizado no presente trabalho, com destaque para FernándezAkarregui et al. (2012) atingindo 420 horas (25200 minutos) de operação.

Além disso, nas Figuras 4 e 5 são apresentadas curvas que mostram em termos percentuais a perda de massa ao longo do tempo $\left(X_{a}\right)$. Nota-se que o comportamento das curvas dos dois materiais é similar, isto é, aumenta com o aumento do tempo, porém, a quantidade de massa perdida foi maior para as partículas de dolomita, aproximadamente, $37,5 \%$, enquanto que para as esferas de alumina a perda teve o valor aproximado de $32 \%$.

Figura 4 - Perfil da redução mássica das esferas de alumina em função do tempo e evolução temporal da perda de massa.

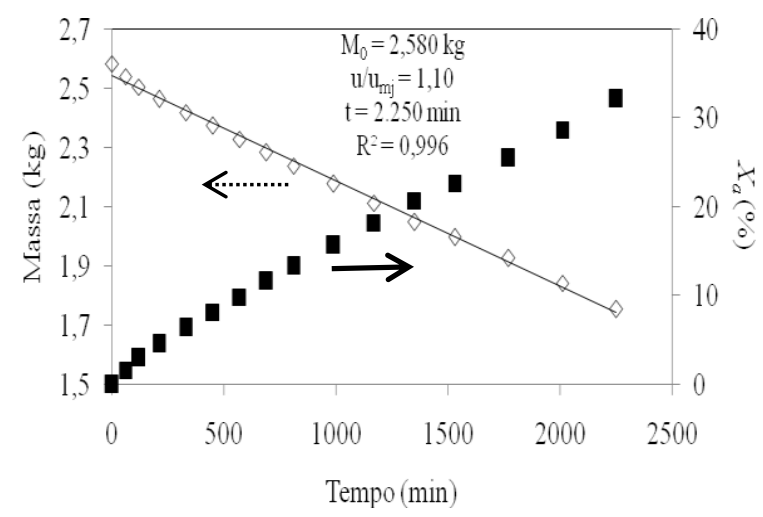

Figura 5 - Perfil da redução mássica das partículas de dolomita em função do tempo e evolução temporal da perda de massa.

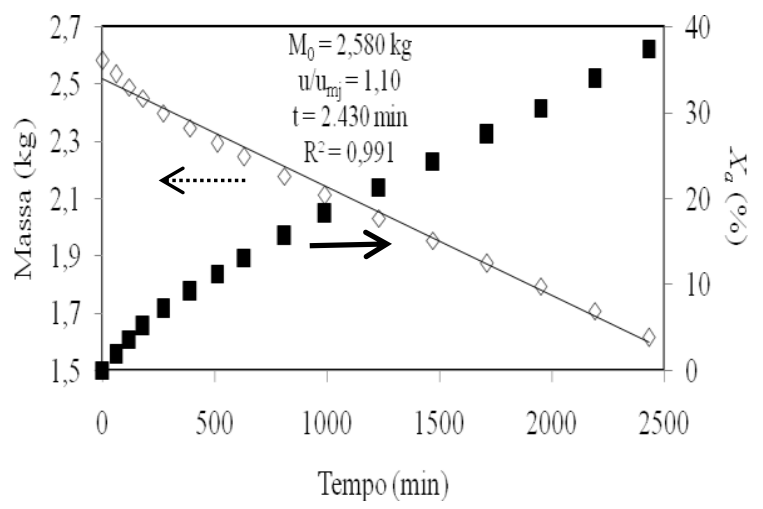

As Figuras 6 (a) e (b) são curvas fluidodinâmicas referentes à alumina na condição inicial $(\mathrm{t}=0)$ e ao final da atrição $(t=2250$ minutos $)$ para as esferas de alumina e na condição inicial $(\mathrm{t}=0) \mathrm{e}$ $(\mathrm{t}=2430$ minutos $)$ ao final da atrição para as partículas de dolomita, respectivamente.

Visualmente, nota-se que a queda de pressão máxima e a velocidade de mínimo jorro tendem a se tornar menores ao final do processo. Isso era esperado, pois com a perda de massa devido o desgaste o leito de partículas se tornou "mais leve" e com isso a energia necessária para romper a coluna de sólidos e mantê-lo em jorro estável se tornou menor. Esse efeito ocorreu também com as partículas de dolomita como mostram as Figuras 7 (a) e (b).

É válido mencionar que para comparar a cinética de atrição da alumina com a dolomita, buscou-se utilizar condições operacionais iguais. Contudo, isso não foi possível, pois as diferentes propriedades físicas e morfológicas dos materiais sólidos influenciaram na fluidodinâmica do leito de jorro. 
Figura 6 - Curvas da queda de pressão em função da velocidade do ar para as esferas de alumina: (a) inicial e (b) final.

(a)

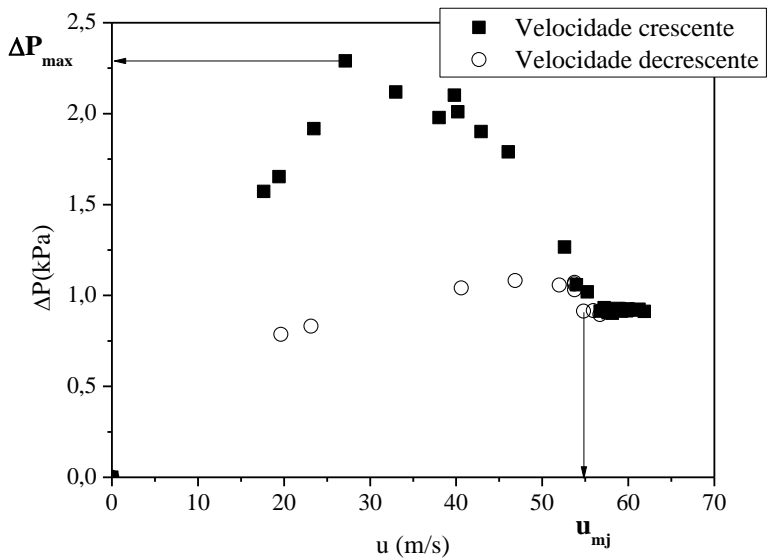

(b)

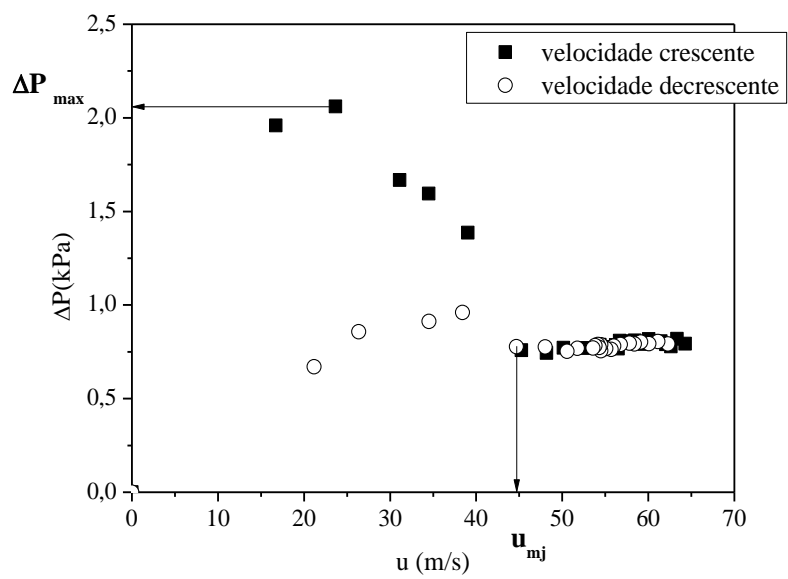

Figura 7 - Curva da queda de pressão em função da velocidade do ar para as partículas de dolomita: (a) inicial e (b) final.

(a)

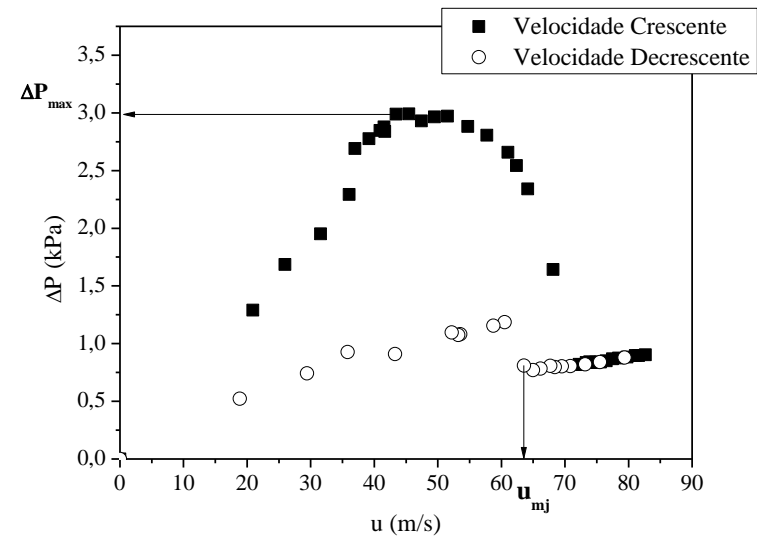

(b)

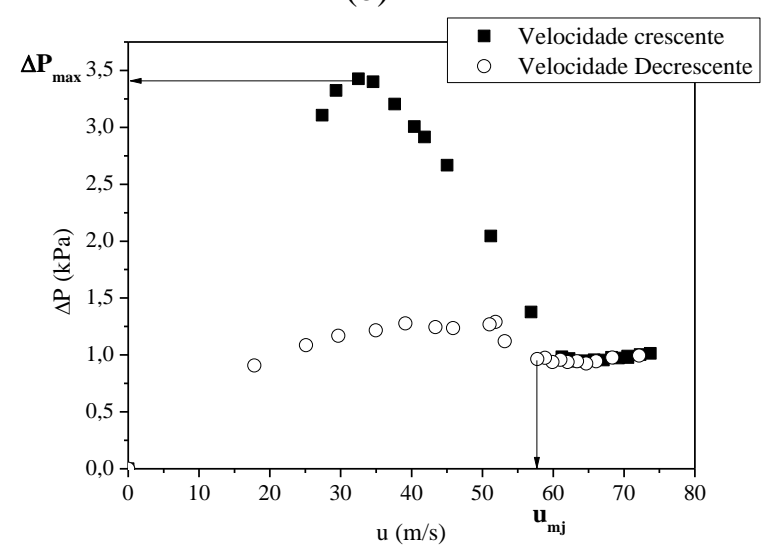

É possível verificar visualmente através das Figuras 6 e 7, apresentadas anteriormente que o efeito da atrição sobre o desempenho do leito de jorro foi significativo na velocidade de mínimo jorro e o qual pode ser observado facilmente na Figura 8. Notase que a velocidade de mínimo jorro diminui ao longo do tempo para ambos os materiais particulados.

Figura 8 - Dados da velocidade de mínimo jorro $\left(\mathrm{u}_{\mathrm{mj}}\right)$ para as esferas de alumina e as partículas de dolomita em função do tempo.

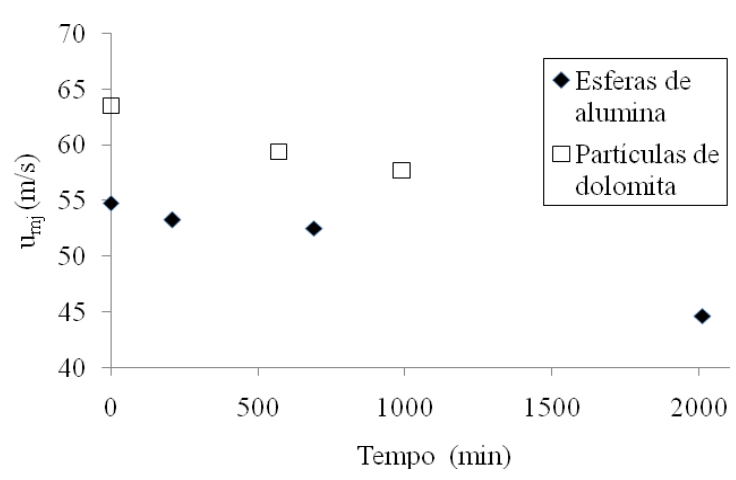

O efeito da velocidade do ar de entrada na atrição foi analisado a partir da comparação dos resultados obtidos para as relações $\mathrm{u} / \mathrm{u}_{\mathrm{mj}}=1,10 \mathrm{e} \mathrm{u} / \mathrm{u}_{\mathrm{mj}}=1,20$, como são apresentadas nas Figuras 9 e 10 os dados de cinética de atrição e da perda de massa $\left(X_{a}\right)$. 
Analisando a Figura 9, observa-se através da cinética de atrição que a massa das esferas de alumina decresce à medida que aumenta o tempo para as duas condições operacionais, além disso, verifica-se que para a maior velocidade do ar de entrada $(\mathrm{u} / \mathrm{umj}=1,20)$ a redução da massa se torna mais acentuada. Neste caso, fica caracterizado o efeito da velocidade do ar no processo de transferência de quantidade de movimento da fase particulada. Ao utilizar a maior velocidade do ar, o arraste das esferas de alumina e o número de colisões entre os sólidos e dos sólidos contra a parede do leito de jorro também se tornaram maiores e consequentemente a atrição.

$\mathrm{Na}$ Figura 10 são apresentadas as curvas da perda de massa para as esferas de alumina. Nota-se que com o aumento do desgaste a perda de massa também foi maior. Este comportamento é coerente e concorda com o que era esperado. Quantitativamente, a diferença pode ser considerada significativa, sendo que, para o intervalo de tempo verificado, a perda mássica alcançou, em torno, de $25 \%$ para $u / u_{m j}=1,20$.

Figura 9 - Perfil da redução mássica das esferas de alumina em função do tempo parametrizada nas velocidades do ar de entrada.

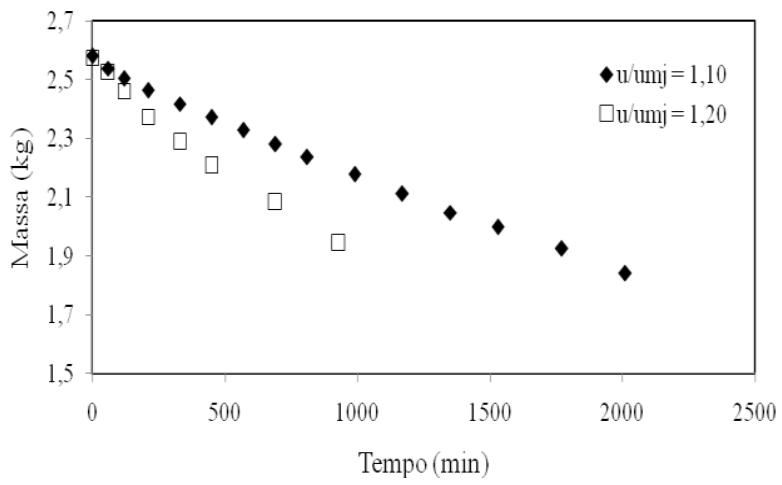

Figura 10 - Evolução temporal da perda de massa parametrizada nas velocidades do ar de entrada.

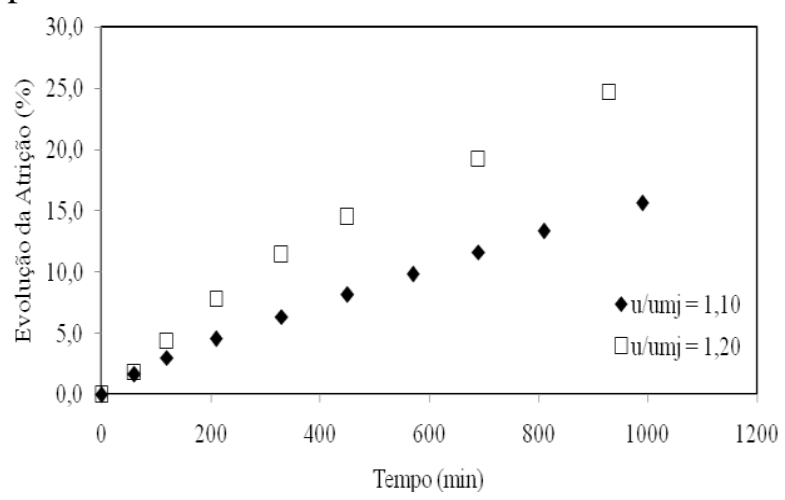

A cinética de atrição das esferas de alumina e das partículas de dolomita foi também analisada do ponto de vista numérico para $\mathrm{u} / \mathrm{umj}=1,10$, como mostram as Figuras 11 e 12.

No caso das esferas de alumina, os parâmetros estimados para o modelo de Gwyn (1969) foram $k_{p}$ e m, cujo seus resultados são $3,61 \times 10^{-004} \mathrm{~s}^{-\mathrm{m}}$ e 0,91 , respectivamente. Para o modelo de Lee et al. (1993) os parâmetros estimados foram $\mathrm{k}_{0}$ e $\mathrm{k}_{1}$, cujo valores são, $8,99 \times 10^{-004} \mathrm{~s}^{-1}$ e $-4,42 \mathrm{~m}^{2} / \mathrm{s}^{2}$.

Para as partículas de dolomita os parâmetros estimados para o modelo de Gwyn (1969) foram $k_{p}$ e m, cujo seus resultados são $8,43 \times 10^{-004} \mathrm{~s}^{-\mathrm{m}}$ e 0,82 , respectivamente. Para o modelo de Lee et al. (1993) os parâmetros estimados foram $\mathrm{k}_{0}$ e $\mathrm{k}_{1}$, cujo valores são, $0,001 \mathrm{~s}^{-1}$ e $4,42 \mathrm{~m}^{2} / \mathrm{s}^{2}$.

Ambos os modelos apresentaram resultados simulados coerentes com o comportamento fenomenológico, entretanto, os resultados preditos pelo modelo proposto por Gwyn (1969) apresentaram melhores concordâncias com os resultados experimentais, tanto para a alumina quanto para a dolomita, como mostram as Figuras 11 e 12.

Deve-se levar em conta que o modelo proposto por Lee et al. (1993), possui um termo exponencial, o que pode ter influenciado para o decaimento exponencial 
da massa ao longo do tempo, como mostram as Figuras 11 e 12.

Figura 11 - Dados experimentais e simulados da redução mássica das esferas de alumina em função do tempo.

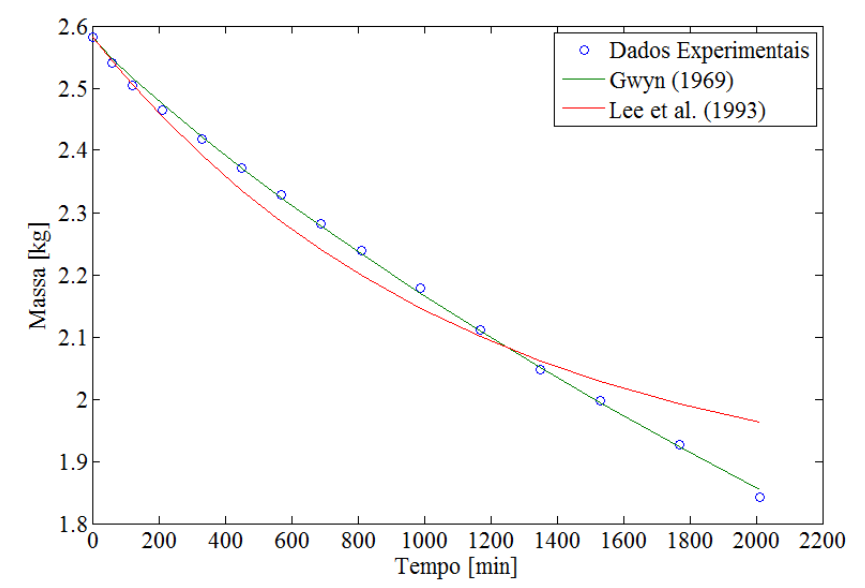

Figura 12 - Dados experimentais e simulados da redução mássica das partículas de dolomita em função do tempo.

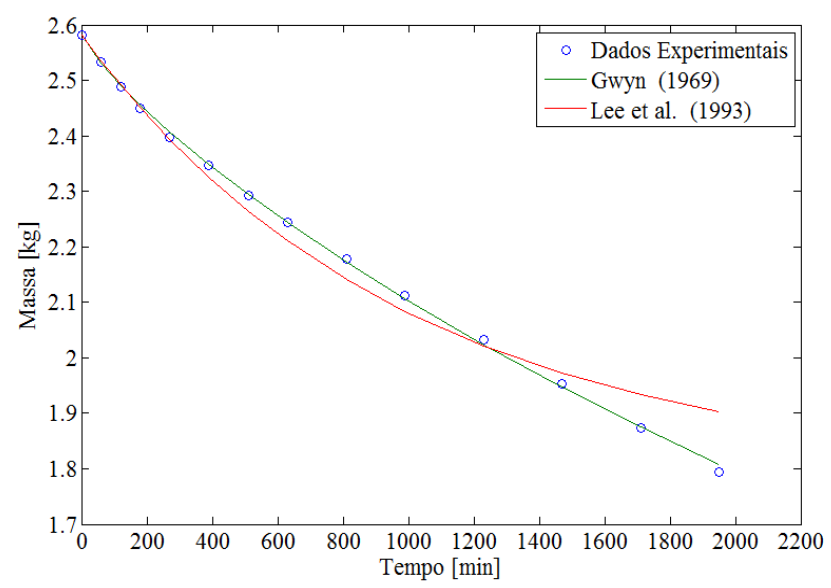

\section{CONCLUSÕES}

Assim, pode-se concluir que foi possível analisar a cinética de atrição dos materiais particulados no leito de jorro tanto por ensaios experimentais quanto pelos modelos numéricos utilizados.

Pela análise granulométrica constatouse que esferas de vidro não se desgastaram, no entanto, o desgaste foi observado para as esferas de alumina e as partículas de dolomita.

Foi possível observar, a partir da cinética de atrição que as esferas de alumina e as partículas de dolomita apresentaram reduções lineares da massa em função do tempo. Além disso, foi visto também que a redução das esferas de alumina foi mais acentuada ao utilizar a maior velocidade do ar de entrada.

A atrição dos dois materiais utilizados influenciou no desempenho fluidodinâmico do leito de jorro, alterando parâmetros fluidodinâmicos, principalmente na velocidade de mínimo jorro.

Os modelos numéricos utilizados apresentaram resultados coerentes, contribuindo para a compreensão da atrição no leito de jorro, contudo, o modelo proposto por Gwyn (1969) forneceu previsões aceitáveis em relação aos resultados observados nesse trabalho.

\section{NOMENCLATURA}

\begin{tabular}{ccc}
$\mathrm{dp}$ & Diâmetro da partícula & $\mathrm{mm}$ \\
$\mathrm{dp}_{\mathrm{s}}$ & Diâmetro médio de Sauter & $\mathrm{mm}$ \\
$\mathrm{K}_{0}$ & Constante cinética da Eq. 5 & $\mathrm{s}^{-1}$ \\
$\mathrm{~K}_{1}$ & Constante cinética da Eq. 5 & $\mathrm{s}^{-1}$ \\
$\mathrm{~K}_{\mathrm{a}}$ & Constante de atrição do modelo & $\mathrm{s}^{-1}$ \\
\multicolumn{3}{c}{ de Lee et al. } \\
$\mathrm{K}_{\mathrm{p}}$ & Constante da Eq. 3 & $\mathrm{s}$ \\
$\mathrm{m}$ & Expoente dependente do tempo & $(-)$ \\
& da atrição da Eq. 3 \\
$\mathrm{M}_{0}$ & Massa inicial do sólido & $\mathrm{kg}$ \\
$\mathrm{M}_{\mathrm{i}}$ & Massa do sólido & $\mathrm{kg}$ \\
$\mathrm{R}_{\mathrm{t}}$ & Taxa de atrição & $\mathrm{kg} / \mathrm{s}$ \\
$\mathrm{t}$ & Tempo & $\mathrm{s}$ \\
$\mathrm{u}$ & Velocidade do ar de entrada & $\mathrm{m} / \mathrm{s}$ \\
$\mathrm{u}_{\mathrm{mj}}$ & Velocidade de mínimo jorro & $\mathrm{m} / \mathrm{s}$ \\
$\mathrm{W}_{\mathrm{e}}$ & Massa dos sólidos no tempo t & $\mathrm{kg}$ \\
$\mathrm{W}_{\mathrm{min}}$ & Massa mínima dos sólidos & $\mathrm{kg}$ \\
$\mathrm{x}$ & Fração mássica & $(-)$
\end{tabular}


$X a$

$\rho_{\text {ap }}$

$\rho_{\text {real }}$
Perda mássica
Massa específica aparente
Massa específica real

(\%)

$\mathrm{kg} / \mathrm{m}^{3}$

$\mathrm{kg} / \mathrm{m}^{3}$

Letra grega

$\Phi$

Esfericidade
WERTHER. J., REPPENHAGEN, J. Handbook of fluidization and fluid-particle systems. New York: Marcel Dekker, Inc., 2003.

\section{REFERÊNCIAS}

BENROSE, C.R., BRIDGWATER, J. A review of attrition and attrition test methods. Powder Technology, v. 49, p. 97126, 1987.

FERNÁNDEZ-AKARREGUI, A. R., MAKIBAR. J., ALAVA, I., DIAZ, L., CUEVA, F., AGUADO, R., LOPEZ, G., OLAZAR,M. Sand attrition in conical spouted beds. Particuology. v. 10, p. 592599, 2012.

GWYN, J. E. On the particle size distribution function and the attrition of craking catalysts. AICHE Journal, v. 15, p. 35-39, 1969.

LEE, S. K., JIANG, X., KEENER. T. C., KHANG, S. J. Attrition of lime sorbents during fluidization in a circulating fluidized bed absorber. Ind. Eng. Chem. Res., v. 32, p. 2758 - 2766, 1993.

MATHUR, K. B., EPSTEIN, N. Spouted beds. New York: Academic Press., 1974.

PAPADOPOULOS. D. G., Impact breakage of particulate solids. 1998. 204p. Thesis (Doctor Philosophy) - University of Furrey, 1998.

RAY, Y.C e JIANG, T.S., 1987. Particle attrition phenomena in a fluidized bed. Powder Technology, v. 49, p. 193-206, 1987. 